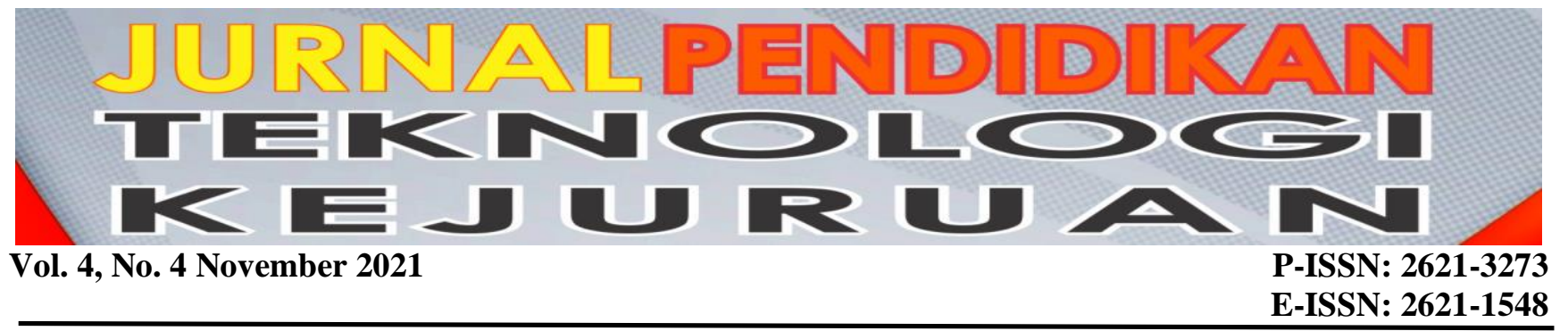

\title{
Productivity Analysis Using Method Murvin E. Mundel Case Study PT. Johan Sentosa
}

\author{
Riko Ervil ${ }^{*}$, Dian Yudistira Rhamadan ${ }^{2}$ \\ ${ }^{1,2}$ Industrial Engineering, Sekolah Tinggi Teknologi Industri Padang \\ "Corresponding author, e-mail: rikopdg01@gmail.com
}

\begin{abstract}
Productivity is an alternative to evaluating the performance that has been done even one of the most effective ways in assessing the efficiency of using several inputs in producing certain outputs. the study was conducted at PT Johan Sentosa. The company is engaged in processing palm oil into CPO (crude palm oil) and kernels. In this case, the company must replace an important process called the sterilizer system from the horizontal sterilizer to the vertical sterilizer. This change will certainly have an impact on company productivity. In this study, the method used is Marvin $\mathbf{E}$ Mundel where partial productivity will be identified in the form of depreciation value index, material, labor, energy, and maintenance, and know the total production value index. The results of this study obtained a partial productivity index on the declining workforce, other partial productivity such as depreciation, material, labor, energy, and maintenance have increased while the index value of total productivity increased after a change in the sterilizer system.
\end{abstract}

Keywords: Partial productivity, total productivity, productivity index

\section{INTRODUCTION}

PT Johan Sentosa, This company is engaged in processing palm oil into CPO (crude palm oil) and kernel. Behind a good processing process, of course, good machine maintenance is also carried out. There are times when the machine enters a period of having to be replaced because its lifetime has expired, so it is no longer economical to continue operating. The phase where we change machines will cause an increase in maintenance costs, the swelling affects the company's productivity because one of the inputs to the production value is maintenance costs. This occurred at the company PT Johan prosperous, where they have to replace an important process called system sterilizer of sterilizerhorizontal to sterilizervertical. The sterilizer itself is a combined process of material handling and boiling of palm fruit, where all raw materials that are ready to be processed are brought by the method lorry or conveyor to the boiling either in the form of the old horizontal or the new vertical at PT. This is Johan Sentosa. After the change in the system, the sterilizer author is interested in researching how the company's productivity is. Application productivity analysis with the method of Marvin E. Mundel

As a tool to analyze the success of the company. The study has the objective to calculate an index value of the company after the partial productivity system changes sterilizer and the index value of the total productivity of the company after the change sterilizes system.

\section{METHOD}

The type of research conducted by the author is descriptive quantitative research, namely processing data in the form of numbers in a company or industry which later data will be collected and processed to provide useful information using mathematical formulations. So this research includes the process of collecting, presenting, and processing data, as well as analysis and interpretation at PT. John Sentosa.

In productivity, there are input and output variables used for output and input, namely depreciation costs, energy costs, material costs, maintenance costs, labor costs, while the output variables are the amount of production, CPO prices, and kernel prices.

The types of data used in this study are primary data and secondary data, namely in the form of what costs are used, and how much profit is obtained.

To perform productivity calculations using the Murvin E.Mundel Method, it is done after the data that can be collected,

1. Deflator Calculation

2. Constant Price Calculation

3. Output Aggregate Calculation

4. Partial Productivity Index Calculation 


\section{RESULT AND DISCUSSION}

The stages in the processing of the Murvin E. Mundel method are as follows:

1. Partial Costs

Table 1. Partial Cost Data

\begin{tabular}{|l|c|c|c|c|c|}
\hline \multicolumn{7}{|c|}{ Biaya } \\
\hline Periode & Depresiasi & Material & Tenaga Kerja & Energi & Maintenance \\
\hline Januari & 26.891 .456 & 17.695 .702 .260 & 308.739 .297 & 238.650 .879 & 280.489 .792 \\
\hline Februari & 24.766 .014 & 15.429 .708 .800 & 321.875 .148 & 156.765 .189 & 200.963 .680 \\
\hline Maret & 26.367 .796 & 16.293 .136 .500 & 337.713 .865 & 135.608 .948 & 228.956 .428 \\
\hline April & 37.503 .261 & 17.459 .008 .000 & 368.364 .088 & 142.193 .069 & 375.860 .437 \\
\hline Mei & 31.173 .142 & 20.505 .223 .200 & 329.821 .715 & 190.926 .900 & 277.224 .313 \\
\hline Juni & 37.377 .583 & 22.982 .365 .080 & 387.276 .201 & 151.822 .238 & 204.969 .299 \\
\hline Juli & 34.530 .724 & 25.654 .392 .510 & 422.035 .165 & 114.922 .147 & 181.825 .713 \\
\hline Agustus & 37.583 .350 & 22.837 .440 .360 & 408.850 .929 & 106.359 .595 & 148.730 .090 \\
\hline September & 37.333 .842 & 21.763 .680 .460 & 402.664 .524 & 123.836 .556 & 176.107 .949 \\
\hline Oktober & 41.307 .494 & 22.573 .912 .000 & 421.835 .658 & 142.976 .071 & 203.120 .179 \\
\hline Nopember & 35.732 .060 & 15.891 .331 .280 & 400.145 .035 & 213.725 .724 & 209.709 .384 \\
\hline Desember & 36.224 .916 & 14.884 .382 .280 & 566.770 .403 & 185.805 .990 & 238.310 .482 \\
\hline
\end{tabular}

Source: Mill Manager Report PT Johan Sentosa 2018

2. Price Index Data

Table 2. BPS Riau 2018 Price Index Data

\begin{tabular}{|c|c|c|c|c|c|}
\hline \multirow{2}{*}{ Periode } & \multicolumn{5}{|c|}{ Indeks Harga } \\
\cline { 2 - 6 } & Material & enaga Ker & Depresias & Energi & Laintenenc \\
\hline 18-Jan & 440,2 & 513,8 & 304,4 & 170,9 & 357,3 \\
\hline 18-Feb & 435,7 & 514,1 & 320,6 & 171 & 357,3 \\
\hline 18-Mar & 440,9 & 520 & 321,6 & 171,4 & 362,9 \\
\hline 18-Apr & 440,8 & 520 & 321,7 & 170,7 & 362,9 \\
\hline Mei-18 & 449,1 & 522,2 & 323,8 & 171,1 & 362,9 \\
\hline 18-Jun & 464,6 & 533,3 & 325,7 & 172,3 & 380,5 \\
\hline 18-Jul & 462,6 & 534,6 & 326,7 & 172,4 & 380,5 \\
\hline Agust-18 & 465,6 & 546,5 & 327,6 & 173 & 374,3 \\
\hline 18-Sep & 474,7 & 547,1 & 327,6 & 173,8 & 376,8 \\
\hline Okt-18 & 477,9 & 547,8 & 331,7 & 174 & 376,8 \\
\hline Nop-18 & 485,3 & 547,8 & 331,8 & 175,1 & 376,8 \\
\hline Des-18 & 497,3 & 560,2 & 334,4 & 176,5 & 376,8 \\
\hline
\end{tabular}

Source: BPJS Riau in figures for the 2018 period

3. Production Data

Table 3. Production Data of PT Johan Sentosa 2018

\begin{tabular}{|l|c|c|}
\hline \multicolumn{1}{|c|}{ Periode } & CPO $(\mathbf{K g})$ & Kernel $(\mathbf{~ k g})$ \\
\hline Januari & 2.559 .943 & 442.816 \\
\hline Februari & 2.276 .677 & 408.984 \\
\hline Maret & 2.466 .249 & 540.850 \\
\hline April & 2.523 .943 & 573.120 \\
\hline Mei & 3.154 .600 & 683.550 \\
\hline Juni & 3.631 .275 & 745.146 \\
\hline Juli & 4.454 .243 & 932.226 \\
\hline Agustus & 4.163 .402 & 547.640 \\
\hline September & 3.902 .599 & 648.792 \\
\hline Oktober & 4.601 .788 & 827.978 \\
\hline Nopember & 3.691 .841 & 745.066 \\
\hline Desember & 3.580 .739 & 809.532 \\
\hline
\end{tabular}

Source: Mill Manager Report PT Johan Sentosa 2018

\section{Average Price of CPO and Palm Kernel Oil}

Table 4. Average Price of CPO and Kernel Oil 2018

\begin{tabular}{|l|c|r|}
\hline \multicolumn{3}{|c|}{ Price CPO and Kernel } \\
\hline \multicolumn{1}{|c|}{ Period } & Cpo & \multicolumn{1}{c|}{ Kernel } \\
\hline January & 7.779 & 7.204 \\
\hline February & 7.843 & 6.840 \\
\hline March & 7.925 & 5.748 \\
\hline April & 8.056 & 5.973 \\
\hline May & 7.733 & 5.821 \\
\hline June & 7.534 & 5.344 \\
\hline July & 7.004 & 5.056 \\
\hline Agust & 6.690 & 5.384 \\
\hline September & 6.822 & 5.725 \\
\hline October & 6.545 & 5.190 \\
\hline November & 6.014 & 4.116 \\
\hline December & 5.950 & 4.108 \\
\hline
\end{tabular}

Source: Mill Manager Report PT Johan Sentosa 2018

5. Deflator Calculation

Research month deflator

$$
=\frac{\text { I. H Research month }- \text { I. H Base month }}{\text { I. H Base month }}
$$

January depreciation example:

January deflator

$$
\begin{gathered}
=\frac{\text { I. H Month January }-\mathrm{I} \text {. H Month January }}{\text { I. H January }} \\
\text { January deflator }=\frac{304,4-304,4}{304,4}=0
\end{gathered}
$$

Table 5: Deflator value

\begin{tabular}{|l|c|c|c|c|c|}
\hline \multicolumn{7}{|c|}{ Deflator } \\
\hline \multicolumn{1}{|c|}{ Periode } & Depresiasi & Material & Tenaga Kerja & Energi & Maintenance \\
\hline Januari & 0 & 0 & 0 & 0 & 0 \\
\hline Februari & 0,053 & 0,01 & 0,001 & 0,001 & 0 \\
\hline Maret & 0,057 & 0,002 & 0,012 & 0,003 & 0,016 \\
\hline April & 0,057 & 0,001 & 0,012 & 0,001 & 0,016 \\
\hline Mei & 0,064 & 0,02 & 0,016 & 0,001 & 0,016 \\
\hline Juni & 0,07 & 0,055 & 0,038 & 0,008 & 0,065 \\
\hline Juli & 0,073 & 0,051 & 0,04 & 0,009 & 0,065 \\
\hline Agustus & 0,076 & 0,058 & 0,064 & 0,012 & 0,048 \\
\hline September & 0,076 & 0,078 & 0,065 & 0,017 & 0,055 \\
\hline Oktober & 0,09 & 0,086 & 0,066 & 0,018 & 0,055 \\
\hline Nopember & 0,09 & 0,102 & 0,066 & 0,025 & 0,055 \\
\hline Desember & 0,099 & 0,13 & 0,09 & 0,033 & 0,055 \\
\hline
\end{tabular}

6. Constant price calculation

$$
\text { Constant price }=\frac{\text { Research period value } \times 100}{100+\text { Deflator }}
$$

Example constant price depreciation January

Constant price depreciation January Score depreciation January x 100 
Constant price depreciation January

$$
\begin{aligned}
& =\frac{26.891 .456 \times 100}{100+0} \\
& =26.891 .456
\end{aligned}
$$

Table 6. Constant price values

\begin{tabular}{|l|c|c|c|c|c|}
\hline \multicolumn{7}{|c|}{ Konstan } \\
\hline Periode & Depresiasi & Material & Tenaga Kerja & Energi & Maintenance \\
\hline Januari & 26.891 .456 & 17.695 .702 .260 & 308.739 .297 & 238.650 .879 & 280.489 .792 \\
\hline Februari & 24.766 .014 & 15.429 .708 .800 & 321.875 .148 & 156.765 .189 & 200.963 .680 \\
\hline Maret & 26.367 .796 & 16.293 .136 .500 & 337.713 .865 & 135.608 .948 & 228.956 .428 \\
\hline April & 37.503 .261 & 17.459 .008 .000 & 368.364 .088 & 142.193 .069 & 375.860 .437 \\
\hline Mei & 31.173 .142 & 20.505 .223 .200 & 329.821 .715 & 190.926 .900 & 277.224 .313 \\
\hline Juni & 37.377 .583 & 22.982 .365 .080 & 387.276 .201 & 151.822 .238 & 204.969 .299 \\
\hline Juli & 34.530 .724 & 25.654 .392 .510 & 422.035 .165 & 114.922 .147 & 181.825 .713 \\
\hline Agustus & 37.583 .350 & 22.837 .440 .360 & 408.850 .929 & 106.359 .595 & 148.730 .090 \\
\hline September & 37.333 .842 & 21.763 .680 .460 & 402.664 .524 & 123.836 .556 & 176.107 .949 \\
\hline Oktober & 41.307 .494 & 22.573 .912 .000 & 421.835 .658 & 142.976 .071 & 203.120 .179 \\
\hline Nopember & 35.732 .060 & 15.891 .331 .280 & 400.145 .035 & 213.725 .724 & 209.709 .384 \\
\hline Desember & 36.224 .916 & 14.884 .382 .280 & 566.770 .403 & 185.805 .990 & 238.310 .482 \\
\hline
\end{tabular}

7. Calculation of Total Resources Input Partial (RIP) RIP January Total $=$ January Depreciation Constant Price + January Constant Material Price + January Constant Price of Labor + January Energy Constant Price + Maintenance Constant Price RIP Total January $=$ Rp 26.891.456 +

$$
\begin{aligned}
& \operatorname{Rp} 17.695 .702 .260+\mathrm{Rp} \\
& 308.739 .297+\mathrm{Rp} 238.650 .879 \\
& +\operatorname{Rp} 280.489 .792 \\
= & \operatorname{Rp} 18.550 .473 .684
\end{aligned}
$$

Tabel 7. Value Total Resources Input Partial (RIP)

\begin{tabular}{|l|l|}
\hline Period & \multicolumn{1}{|c|}{$\mathrm{Rp}$} \\
\hline January & 18.550 .473 .684 \\
\hline February & 16.134 .078 .831 \\
\hline March & 17.021 .783 .537 \\
\hline April & 18.382 .928 .855 \\
\hline May & 21.334 .369 .270 \\
\hline June & 23.763 .810 .401 \\
\hline July & 26.407 .706 .259 \\
\hline Agust & 23.538 .964 .325 \\
\hline September & 22.503 .623 .331 \\
\hline October & 23.383 .151 .402 \\
\hline November & 16.750 .643 .483 \\
\hline December & 15.911 .494 .071 \\
\hline
\end{tabular}

8. Calculation of Aggregate Output

Aggregate Output $=($ Amount of palm oil production in the month of research $\mathrm{x}$ selling price of CPO oil per kilogram $)+($ Number of kernel production in the month of research $\mathrm{x}$ selling price of kernel per kilogram)
Agregat Output January $=(2.559 .943 \mathrm{~kg} \times \mathrm{Rp} 7.779$ $/ \mathrm{kg})+(442.816 \mathrm{~kg} \mathrm{x} \quad \mathrm{Rp} 7.204 \mathrm{~kg})$

$=\operatorname{Rp} 23.103 .843 .061$

Table 8. Score Output Agregat

\begin{tabular}{|l|c|}
\hline \multicolumn{1}{|c|}{ Period } & $\mathrm{Rp}$ \\
\hline January & 23.103 .843 .061 \\
\hline February & 20.653 .428 .271 \\
\hline March & 22.653 .829 .125 \\
\hline April & 23.756 .130 .568 \\
\hline May & 28.373 .466 .350 \\
\hline June & 31.340 .086 .074 \\
\hline July & 35.910 .852 .628 \\
\hline Agust & 30.801 .653 .140 \\
\hline September & 30.337 .864 .578 \\
\hline October & 34.415 .908 .280 \\
\hline November & 25.269 .423 .430 \\
\hline December & 24.630 .954 .506 \\
\hline
\end{tabular}

9. Partial productivity index calculation

January:

Example of depreciation productivity index

IP Depreciation

AOMP

$=\frac{\overline{\text { AOBP }}}{\text { RIMP }} \times 100$

$\overline{\mathrm{RIBP}}$

IP Depreciation

Output aggregate depreciation month January

$=\frac{\text { Output agregat depreciation month January }}{\text { Input depration period month January }} \times 100$

Input depreciation period month January

Input depreciation period month January

IP Depreciation

23.103.843.061

$\frac{23.103 .843 .061}{26.891 .456} \times 100=100$

Table 9. Partial Productivity Index Value

\begin{tabular}{|l|c|c|c|c|c|}
\hline \multicolumn{7}{|c|}{ Nilai Indeks Parsial } \\
\hline Periode & Depresiasi & Material & Tenaga Kerja & Energi & Maintenance \\
\hline Januari & 100 & 100 & 100 & 100 & 100 \\
\hline Februari & 97,07 & 102,52 & 85,75 & 136,09 & 124,77 \\
\hline Maret & 100 & 106,49 & 89,64 & 172,56 & 120,12 \\
\hline April & 73,73 & 104,22 & 86,18 & 172,57 & 76,73 \\
\hline Mei & 105,94 & 105,98 & 114,96 & 153,51 & 124,26 \\
\hline Juni & 97,59 & 104,45 & 108,14 & 213,23 & 185,63 \\
\hline Juli & 121,05 & 107,21 & 113,71 & 322,78 & 239,77 \\
\hline Agustus & 95,39 & 103,3 & 100,67 & 299,14 & 251,42 \\
\hline September & 94,58 & 106,77 & 100,68 & 253,06 & 209,14 \\
\hline Oktober & 96,98 & 116,77 & 109,02 & 248,64 & 205,7 \\
\hline Nopember & 82,31 & 121,79 & 84,39 & 122,13 & 146,29 \\
\hline Desember & 79,14 & 126,75 & 58,07 & 136,93 & 125,48 \\
\hline
\end{tabular}


10.Total Productivity Index Calculation

Table 10. Index Value Produktivitas Total

\begin{tabular}{|l|l|}
\hline Period & Nilai \\
\hline January & 100,00 \\
\hline February & 102,78 \\
\hline March & 106,86 \\
\hline April & 103,76 \\
\hline May & 106,78 \\
\hline June & 105,89 \\
\hline July & 109,19 \\
\hline Agust & 105,06 \\
\hline September & 108,24 \\
\hline October & 118,18 \\
\hline November & 121,13 \\
\hline
\end{tabular}

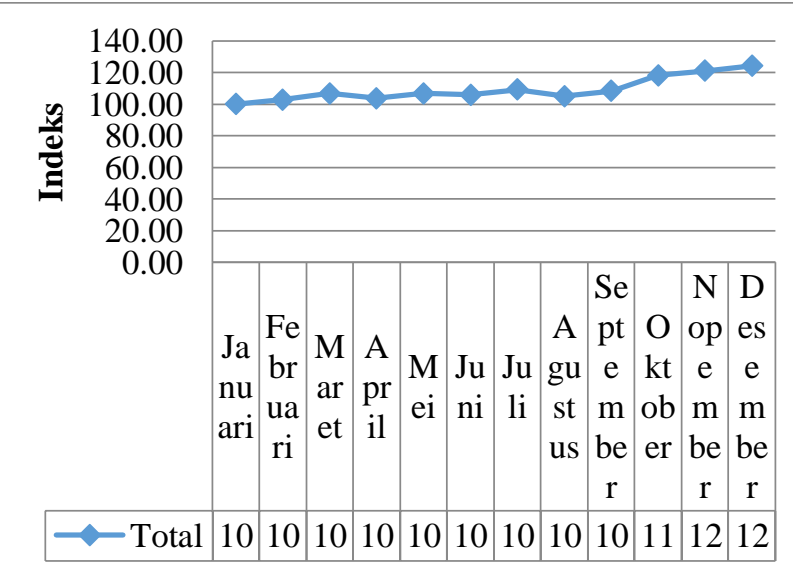

Figure 1 Total Productivity Index

\section{CONCLUSION}

Based on data processing and analysis, the following conclusions can be drawn:

1. The company's partial productivity index after the sterilizer system change can be seen a decrease in labor productivity By setting January as the base period, the index value for February is $85.75 \%$ and then March by $89.64 \%$ followed by April with $86.18 \%$ the highest productivity index was achieved in May at $114.9 \%$, then June at $108.14 \%$ and July at $113.71 \%$, continuing to August with a figure of $100.68 \%$ further to October at $109.02 \%$ and then November with $84.39 \%$ the lowest index occurred in December at $58.07 \%$. due to the adjustment of the new system. While the index of parial productivity values such as depreciation, By setting January as the base period, February got the lowest index of
$102.5 \%$, March of $106.49 \%$, then April with a value of $104.22 \%$, followed by May at $105.98 \%$ and then June at $104,45 \%$ then July at $107.21 \%$ continued to August at $103.30 \%$ then September 106.77\% October at $116.77 \%$ November $121.79 \%$ The highest productivity index was achieved in December at $125.7 \%$. materials, energy, and maintenance have increased.

2. The index of the company's total productivity value after the sterilizer system change has increased. This is in line with the company's goal of increasing productivity.

\section{REFERENCES}

Gaspersz, Vincent. Manajemen Produktivitas Total. Granmedia Pustaka Utama, Jakarta.2000

Sinungan, Muchdarsyah.Produktivitas Apa dan Bagaimana Cetakan 8 Edisi 2. Jakarta: Bumi Aksara.2005.

Summanth. David.J. Productivity Enginering and Management, Mc Graw Hill Book Company, New York 1984

Suprato. Metodologi Penelitian Bisnis Untuk Akuntansi dan Manajemen

Edisi 1. Yogyakarta : BPFE. 2001.

Sahat. Analisis Produktivitas dengan Menggunakan Metode Marvin E.Mundel Pada PT. Coca-cola. Jurnal Departemen teknik Industri, fakultas Teknik, Jurusan teknik Industri, Universitas Sumatra Utara, Medan.2008

Rejeki, Sinulingga, and Tarigan. Analisis Produktivitas dengan Menggunakan Metode Marvin E.Mundel (Studi Kasus pada CV NURYZ BERSAUDARA). Jurnal Teknik Industri.2013 